\title{
Study on the Sound Quality Objective Evaluation of High Speed Train's Door Closing Sound
}

\author{
Zongcai Liu ${ }^{1, a^{*}}$, Zhaojin Sun ${ }^{2, b}$ and Shaoqing $\operatorname{Liu}^{3, \mathrm{c}}$ \\ ${ }^{1}$ National Engineering Research Center for High-speed EMU,CSR Qingdao sifangCo.,Ltd, \\ Qingdao,China \\ ${ }^{2}$ National Engineering Research Center for High-speed EMU,CSR Qingdao sifangCo.,Ltd, \\ Qingdao,China \\ ${ }^{3}$ National Engineering Research Center for High-speed EMU,CSR Qingdao sifangCo.,Ltd, \\ Qingdao,China

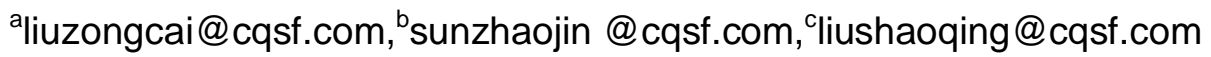

Keywords: High Speed Train; Door Closing Sound; Sound Quality; Objective Evaluation

Abstract. Test the high speed train's door closing sound under different close pressure and then use the linear sound pressure level(SPL), A weight sound pressure level, psychoacoustic parameters of sound quality and specific loudness to analyze the sound. The results are indicated as follows: the frequency of the sound is mainly in the range from $50 \mathrm{~Hz}$ to $3000 \mathrm{~Hz}$. The specific loudness can accurately match with the feeling of human eras. Using the loudness and roughness can have a better characterization to the door closing sound than the sound pressure level.

\section{Introduction}

With the improvement of the train's speed, it brings lots of convenience to people, so more and more people like to choose the high speed train as their vehicle if they want to have a travel. There for the noise of the train has attracted more and more attention as it plays an important part in the comfort index. Because of the safety requirement, the doors will be compacted when the speed higher than the setting speed and a transient sound will be produced during this process, which will influence the comfort level, the auditory sense of human is obviously. The sound is an instant impact and is very short, it will not be good to describe it using the SPL, so other method such as sound quality evaluation should be used to analyze the sound.

Sound quality evaluate method mainly contains two methods including subjective evaluation and objective evaluation. The criterion of the subjective evaluation to judge the sound is good or not is based on the feeling of people. The objective evaluation quantizes the different kinds property of the sound through the psychoacoustic parameters and use the numerical value to estimate the sound. This paper researched the method of the objective evaluation of the high speed train's door closing sound by studying different door's closing sound.

\section{Test the sound}

The train is stopped in the open field with all the equipments being turned off. There is no tall buildings around the train so that they will not make any reflex. The test points is set inside the train. They all are in the face that perpendicular to the $\mathrm{X}$-axis of the train, pointing to the door[1]. The distance between the test points and the floor is $1.6 \mathrm{~m}$. During the test, there is no other people 
express the tester. The air conditioning should be turned off. The door's open and close is controlled by the software. We acquire the No.1 door's different sound under different compact press in the front end of the train and the No.4 door's sound in the back end. The compact press to close the door becomes large gradually. A,B,C, D strands for the compact press of the door respectively and $\mathrm{D}$ is the biggest one. Sift and choose the representative samples of them which will be used to have some analysis. There are 8 samples in all.

\section{Sound character analysis}

In order to give an comprehensive analysis about the door closing sound's character and the distribution rule of the frequency spectrum as well as to improve the sound level effectively, we contrast the different door closing sound's frequency spectrum and use some useful method to give a reasonable objective description.

Time domain and frequency domain analysis. To analyze the selected samples in time domain and frequency domain, we can find out the sound's distribution rule along with the time and frequency, which are shown in figure 1 and 2.

From the figures we can find that during the door closing two crests will turn up. The first crest is bigger than the second one and the duration time is longer while the second one is smaller, shorter and has less energy. The first crest is mainly caused by the different parts of the door's compact mechanism. When the door is closed, the different parts will have a series motions and will collide with each other. The time is short and the different sound overlapped with each other. The second crest is caused by the spring back of the parts. Because of more than once collide will happened when the door is closed and the time is short, some interval will exist among them, which will make the first crest looks like having three small crests. If the compact press is improved, the energy of the noise will increase, the small crests of the first one will become two from three which is shown in figure 3.

The frequency of the No.1 door closing sounds mainly in the range from $50 \mathrm{~Hz}$ to $3000 \mathrm{~Hz}$ and the peak value of the frequency are $500 \mathrm{~Hz}, 1000 \mathrm{~Hz}$ and $1400 \mathrm{~Hz}$. The frequency of the No.4 door closing sounds mainly in the range from $50 \mathrm{~Hz}$ to $5000 \mathrm{~Hz}$ and the peak value of the frequency are $650 \mathrm{~Hz}, 900 \mathrm{~Hz}$ and $1250 \mathrm{~Hz}$. The sound's energy of No.4 door is bigger than that of the No.1 door and the peak is much more obviously.

Contrast the linear sound pressure level and A weight SPL. Take the samples of the No.1 door and No.4 door's closing sound under the A compact press level for example to analyze the frequency domain. From the linear sound pressure level curve in figure 4 we can find that the mainly frequency of No.1 and No.4 door's sound is in the range below 400Hz.The frequency between $50 \mathrm{~Hz}$ and $125 \mathrm{~Hz}$ is much more outstanding and the maximum is $50 \mathrm{~Hz}$. In the range of $50 \mathrm{~Hz}$ to $125 \mathrm{~Hz}$, the SPL of No.4 door closing sound is higher than that of the No.1 door, the difference value is about 3 to $5 \mathrm{~dB}(\mathrm{~A})$. From the A weighted SPL curve in figure 4 we can find that the frequency range from $200 \mathrm{~Hz}$ to $1600 \mathrm{~Hz}$ is highlight and the maximum peak is $500 \mathrm{~Hz}$ because the low-frequency is obviously decayed due to the arithmetic of the A-weighted SPL.

\section{Sound quality objective evaluation analysis}

Sound quality objective parameters is used to describe the different kinds property of the sound including strong, weak, acuity, undulate of the sound and so on. Using the sound quality objective parameters to evaluate the feeling by quantify method can reduce the influence caused by the 
difference of the people's feeling. At present, the loudness, sharpness, roughness and fluctuation have been deeply studied and widely used[2]. We will use them to analyze the sound.
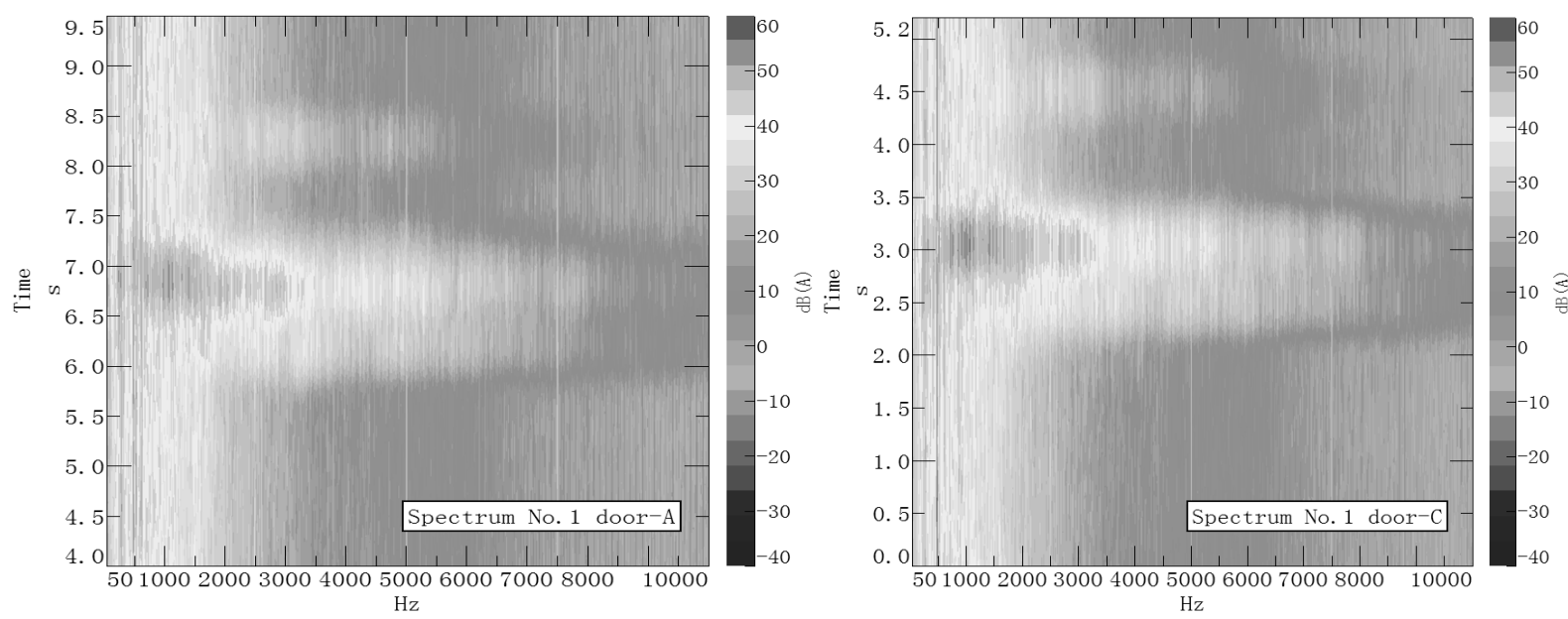

Figure 1 The time domain and frequency domain of No.1 door
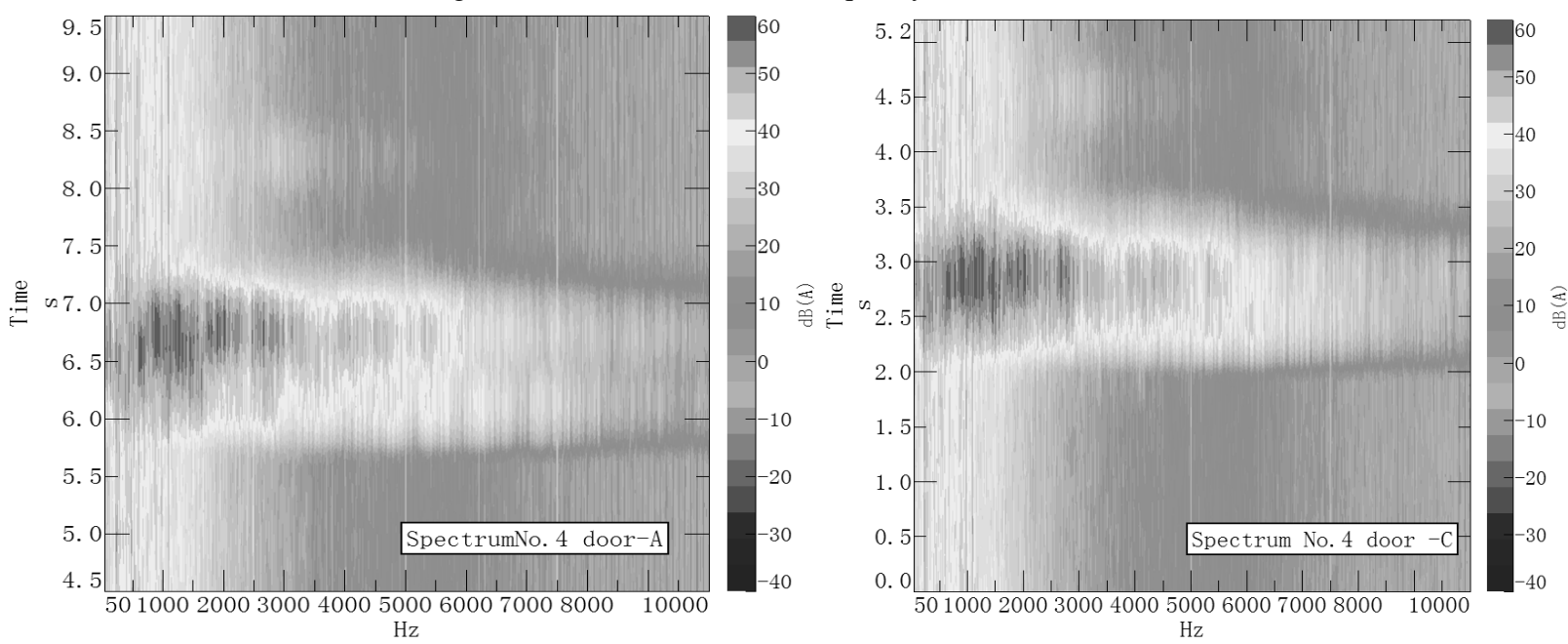

Figure 2 The time domain and frequency domain of No.4 door
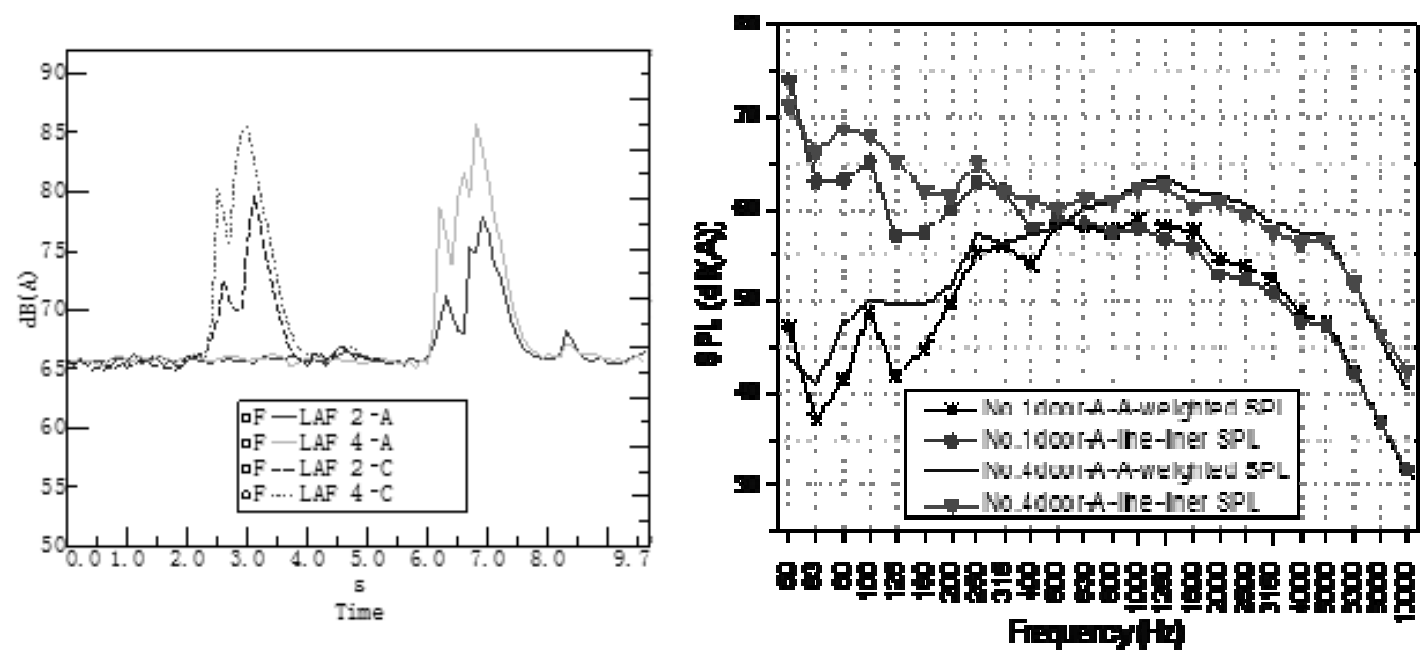

Figure 3 The different door's sound time domain curve

Figure 4 The different weighted SPL's frequency curve

Psychoacoustic parameters of the sound quality analysis. Loudness is a sensation value of the human perception of sound volume. It can be used to describe people's feeling of the sound whether it is loud, small, strong or weak. It takes the shelter of the ear into consideration which will be better than the A weighted SPL to describe the feeling[3]. The unit of the loudness is Sone. There are two 
models to calculate it. The A model was put forward by Stevens and the B model was put forward by Zwicker. As the B model based on the 1/3 Octave to calculate the result and it can be used in condition that the environment is reverberant field as well as the free field, so the reliability is better than A model[4]. Because of B model is adopted in ISO532B, we will use B model in this paper.

The sharpness is the sensation value produced by high frequency components in sounds. It describes the jarring level of the sound. At present Aures sharpness model is widely accepted but not come into being the international standard.

Both the roughness and the fluctuation can be used to describe the feeling caused by the modulation signal. In the low part of the frequency, the change of the sound will make people feel shake and in the high part the change of the sound will make people feel roughness. Also there is no accepted standard at present, we generally use Zwicker model to calculate them.

From the figure 5 8 we can find the tendency in general that the SPL of No. 4 door is higher than that of the No.1 door obversely. With the increase of the compact pressure, the maximum sound pressure level of No.4 door becomes larger but that change of No.1 door closing sound is not obversely and the level is smoothly. And the loudness has the same change tendency as the sound pressure level. Although the sound pressure of No.4 door closing sound is much higher than the No.1 door, the roughness has little difference. The difference of the roughness between the No.4 door closing sound and the No.1 door closing sound is greater, the No.4 door's roughness is 1.5 to 2 times of the No.1 door's.

Analyze the different in figure5 and figure 6, the maximum SPL of No.1 door-B sample is smaller than No.1 door-A sample, but the maximum loudness of the former is larger than the later. No.4 door-D sample's SPL is much higher than No.4 door-C sample and No.4 door-A sample, but the maximum loudness of them is nearly the same. The feeling is not are sounding expression which means that the loudness is not high even if the corresponding SPL is much higher. As is shown in figure 5 and figure 7, the sharpness of the door closing sound has little difference while the difference of the SPL is very obversely especially the sample of No.4 door under different compact pressure. They have the similar sharpness but the different SPL. Compared the sound pressure level of No.4 door-A sample and No.4 door-B sample, the difference of them is inconspicuous, but No.4 door-B closing sound's roughness is lower than that of No.4 door-A.

We have a audition test by replaying the samples, and find that the feeling of No.1 door-A closing sound is better than No.1 door-B, and the feeling of No.4 door-A is better than No.4 door-B. It is evident that the soundness and roughness can be used to evaluate the character of the door closing sound more authentic than the SPL.

Specific loudness analysis. The calculate of the sound is to integral calculate the specific loudness value in all feature band of the frequency. The specific loudness curve can effectively describe the ingredient frequency of the sound that can cause the change of the loudness. The curve is made with the feature band value as the $\mathrm{x}$ axle and the specific loudness value as the $\mathrm{y}$ axle. The more extruder the specific loudness is, the more contribution of the corresponding frequency range make and the more obviously feeling people will have[6]. From the specific loudness curve in figure 9 10 we can found that from 0 to 3 bark, the specific loudness value is higher than others as the corresponding frequency range is within $400 \mathrm{~Hz}$. This result has a consistence with the linear SPL. When the compact pressure is changed, the sound's peak value of the specific loudness and the corresponding frequency has some distinguish. The peak value of the No.1 door closing sound is skewing with the increase of the compact pressure. The main reason is that the impact noise energy is amplified because of the pressure's increase. The specific loudness peak value of No.4 door closing sound is mainly in range of bark 2. Compared the specific loudness curve of No.1 door and No.4 door, there 
exists an prominent peak in the range of bark 5 in the curve of No. 1 door while the curve of No.4 is smoothly at the same band. The main reason is that the spring back sound of No.1 door is louder than that of No.4 door. When using the A weighted SPL to have an analysis, the peak in range of 5 bark as is shown in figure 9 won't appear. So it is reliable to analyze the noise by specific loudness method.

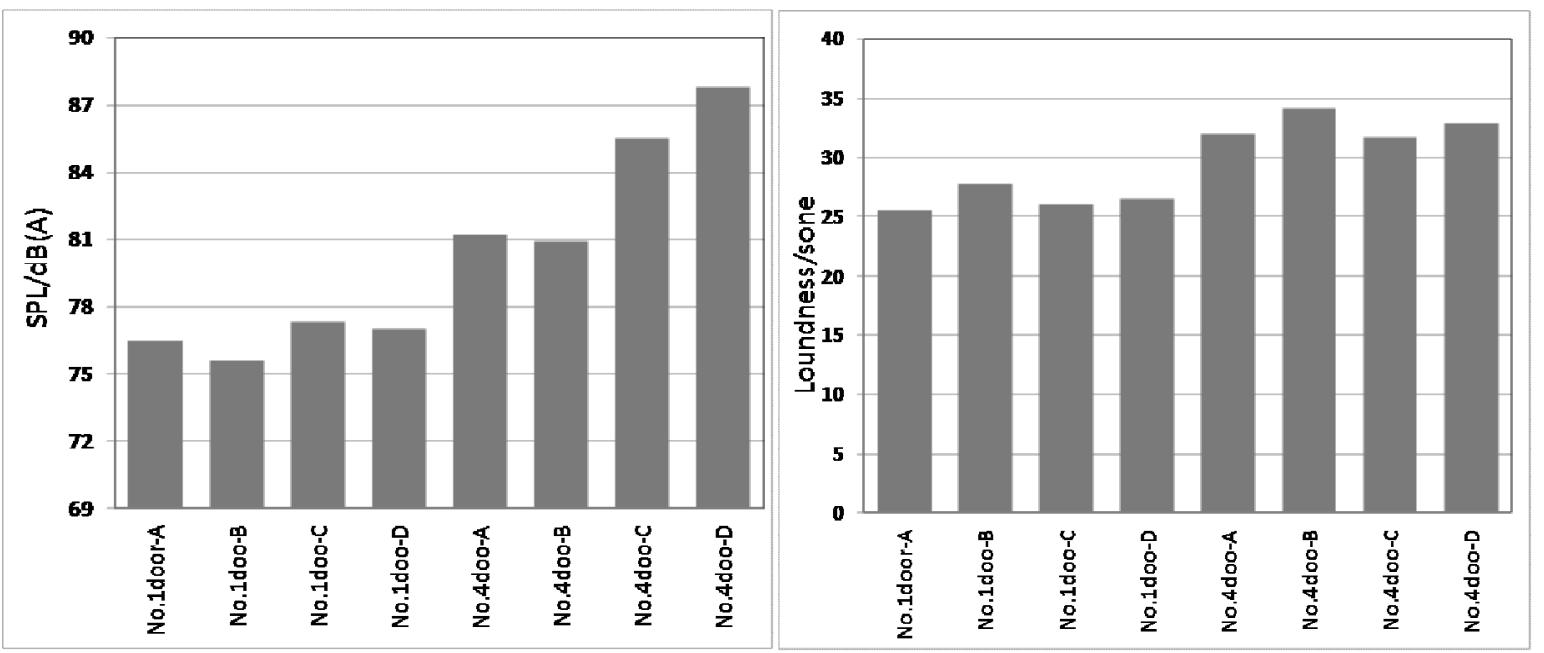

Figure 5 the maximum SPL of the door closing sounds

Figure 6 the loudness of the door closing sounds

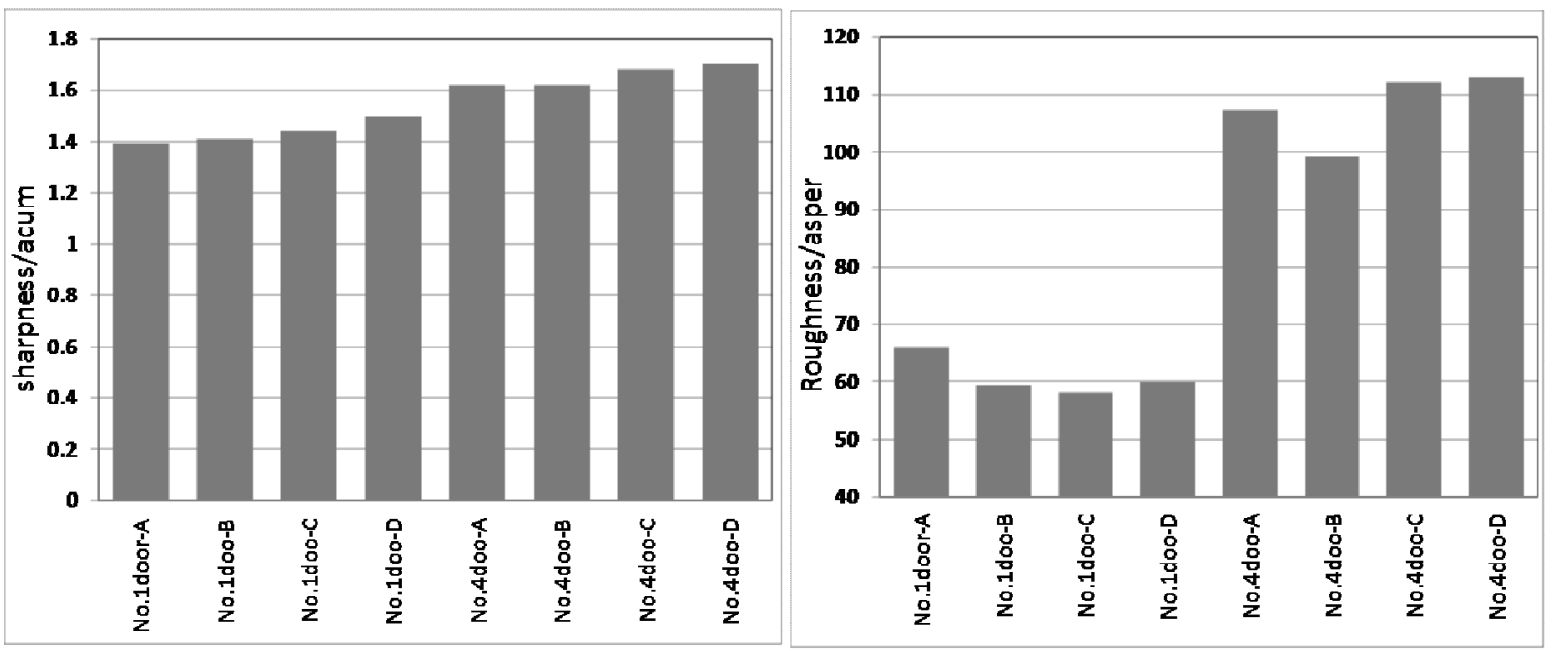

Figure7different door closing sound's sharpness

Figure8different door closing sound's roughness
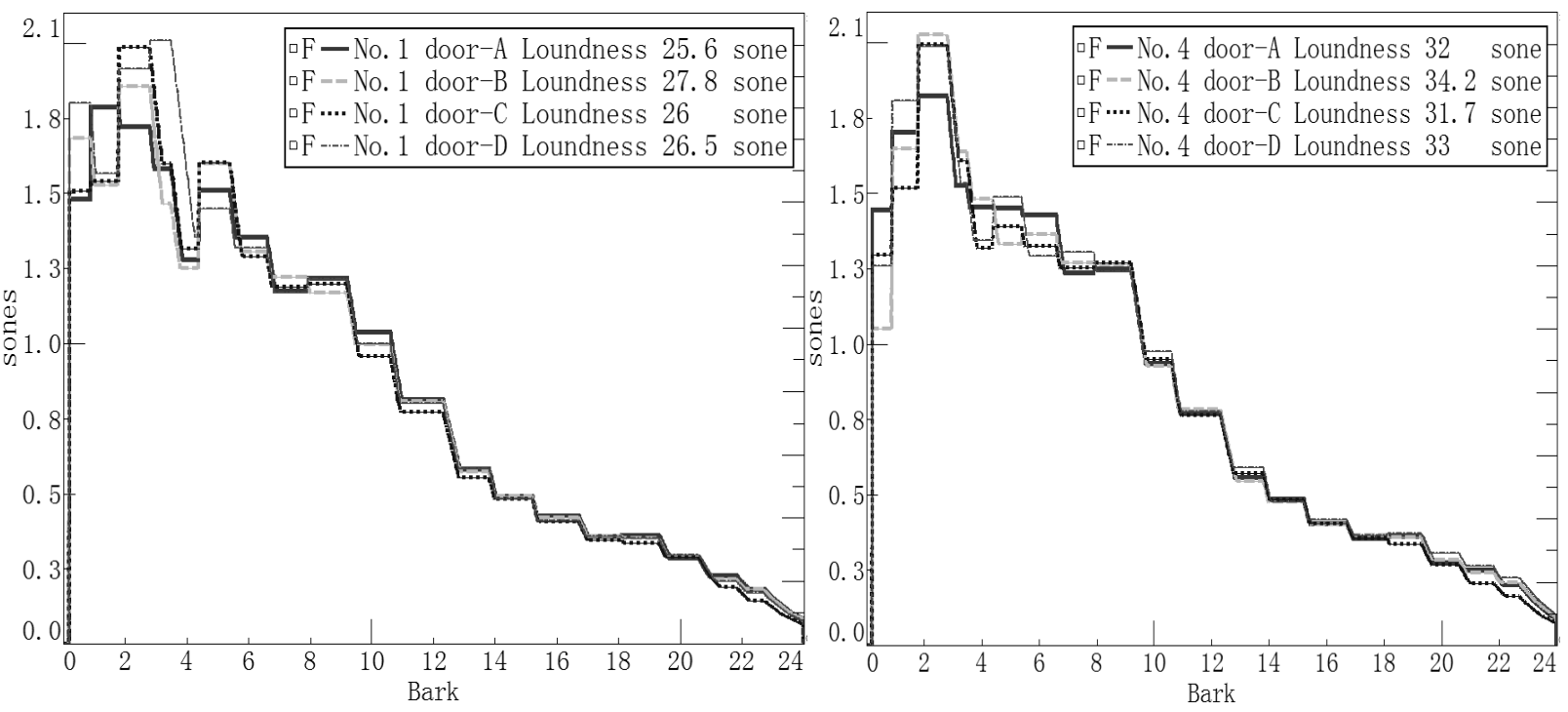

Figure 9specificloudness curve of No.1 door's sound

Figure 10specificloudness curve of No.4 door's sound 


\section{Conclusion}

Using the linear SPL, A weight SPL, time domain and frequency domain to analyze the door closing sound and evaluate the sound with psychoacoustic parameters of sound quality, the conclusions are shown as follows:

(1) When the door is closed, two crests will turn up. The first one has more energy and it give people more obversely feeling. The frequency mainly distribute in the range from $50 \mathrm{~Hz}$ to $3000 \mathrm{~Hz}$.

(2) It will be better to evaluate the door closing sound and to describe people's feeling more effective using the loudness and roughness rather than the A weighted SPL.

(3) The impact feeling caused by the door closing sound is obviously. The main characteristic is that the loudness is higher in the low frequency band than that of others. Therefor some measurement should be taken to solve the problem.

\section{References}

[1]SHEN Zhi-yun,Dynamic. Environment of High-speed Train and Its Distinguished Technology[J],Journal of the China Railway Socity, 28(2006)1-5.

[2]WANG Deng-feng, LIU Zong-wei, et al. Subjective Evaluation Test and Objective Quantificational Description of Vehicle Interior Noise Quality [J]. Joural of Jilin University (Engineering and Technology Edition), 36(2006)41-46.

[3]FANRong-ping. Inter noise evaluation for High Speed Traveling Train Based on Psycho acoustical Loudness Analysis[J].Journal of vibration and shock,24(2005)46-49

[4]Zwicke E, Fastl H. Psychoacoustics: Facts and Models, Second Edited [M].Berlin:Springer-verlag, 1999.15-30.

[5]H.Murata,H.Tanaka,H.Takada,Y.Ohsasa.

Soundqualityevaluationofpassengervehicleinteriornoise,SAE931347(1993)675-681.

[6]BLOMMERM, YANG B, VANDENBRINKK. Detecting and Classifying Secondary Impacts in DoorClosingSound[C]. SAEPaper2005-01-2471. 